\title{
Gravitational Lensing, the Cosmic Microwave Background and Cluster Masses
}

\author{
Scott Dodelson and Chris Vale \\ Particle Astrophysics Center, Fermilab, P.O. Box 500, Batavia, IL 60510 US \\ E-mail: dodelson@fnal.gov, cvale@fnal.gov
}

\begin{abstract}
The deflection of light from distant sources by the foreground mass distribution can be quantified and mined for interesting cosmological information. Within the last decade, observers have moved from making the first detections of this phenomenon to planning ambitious future surveys. These surveys may teach us much about fundamental physics such as dark energy and neutrino mass. After a qualitative review of some of this, we present new results regarding the accuracy with which cluster masses can be determined through a detailed study of the way in which the clusters distort the cosmic microwave background.
\end{abstract}

\section{Introduction}

Gravitational lensing has recently become a powerful tool for extracting cosmological information (see, e.g., [1, 2] for general reviews or [3] for a review of lensing of the $\mathrm{CMB}$. The state of weak lensing in particular seems similar today to the state of the cosmic microwave background (CMB) ten years ago: the first detections of cosmic shear were made several years ago, the theory and the relevant predictions are still new enough that work clearly needs to be done, and there is uncertainty about which class of measurements will prove most useful. The stakes are high. Weak lensing has the potential to measure quantities as important and fundamental as the dark energy equation of state and the neutrino mass. To date, we have probed the cosmic shear field by looking at distortions in the shapes of background galaxies. In the future, we have hopes of using a different probe: the photons in the CMB. After briefly reviewing the success and promise of probing the cosmic shear field, we report results on the accuracy with which cluster masses can be determined when one studies the lensing of the CMB.

\section{Two Point Statistics}

Cosmic shear is a random two-component field on the sky. We are not particularly interested in the shear in a given spot on the sky since no theory makes such definite predictions. Rather we do cosmology by studying the statistics of the cosmic shear. This is another sense in which cosmic shear is similar to the cosmic microwave background 


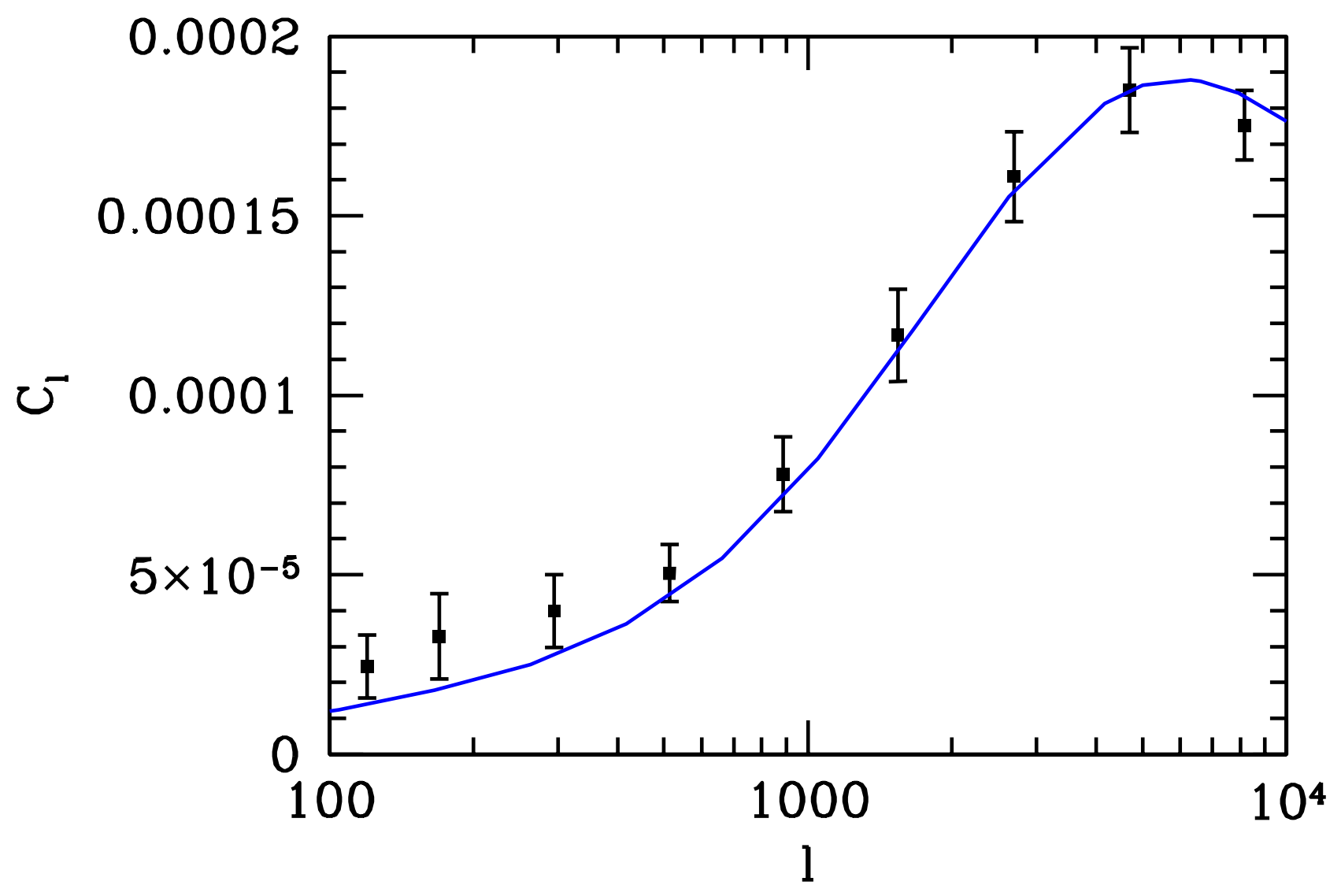

Figure 1. Power spectrum of cosmic shear from ray tracing through numerical simulations (data points with error bars) and via an integration of the power spectrum along the line of sight. Adapted from Ref. [4].

(CMB). We do not care at all where the hot spots and cold spots of the microwave background are. We simply are interested in their statistics.

The means of each of the two shear components are zero, so the first relevant statistic is the two-point function. This situation is again similar to the cosmic microwave background. In fact, we will borrow quite a bit of the notation to discuss cosmic shear.

The power spectrum of the cosmic shear can be written as an integral along the line of sight of the power spectrum of fluctuations in the gravitational potential. Explicitly,

$$
C_{l}=\frac{l^{4}}{4} \int_{0}^{\chi_{s}} d \chi W(\chi) P_{\Phi}(l / \chi ; \chi)
$$

where $\chi$ is the comoving distance to an object, $P_{\Phi}$ is the power spectrum of the gravitational potential, and $W$ is a weighting function which peaks halfway in between the source $\left(\right.$ at $\chi_{s}$ ) and us. As Fig. 1 shows, we can predict quite accurately - given a model - the power spectrum of cosmic shear.

The first detections of cosmic shear using background galaxies as probes were reported in 2000, and since then over a dozen groups have measured this small signal, moving to increasingly large surveys (for a review, see, e.g., [5]). Currently, the surveys 


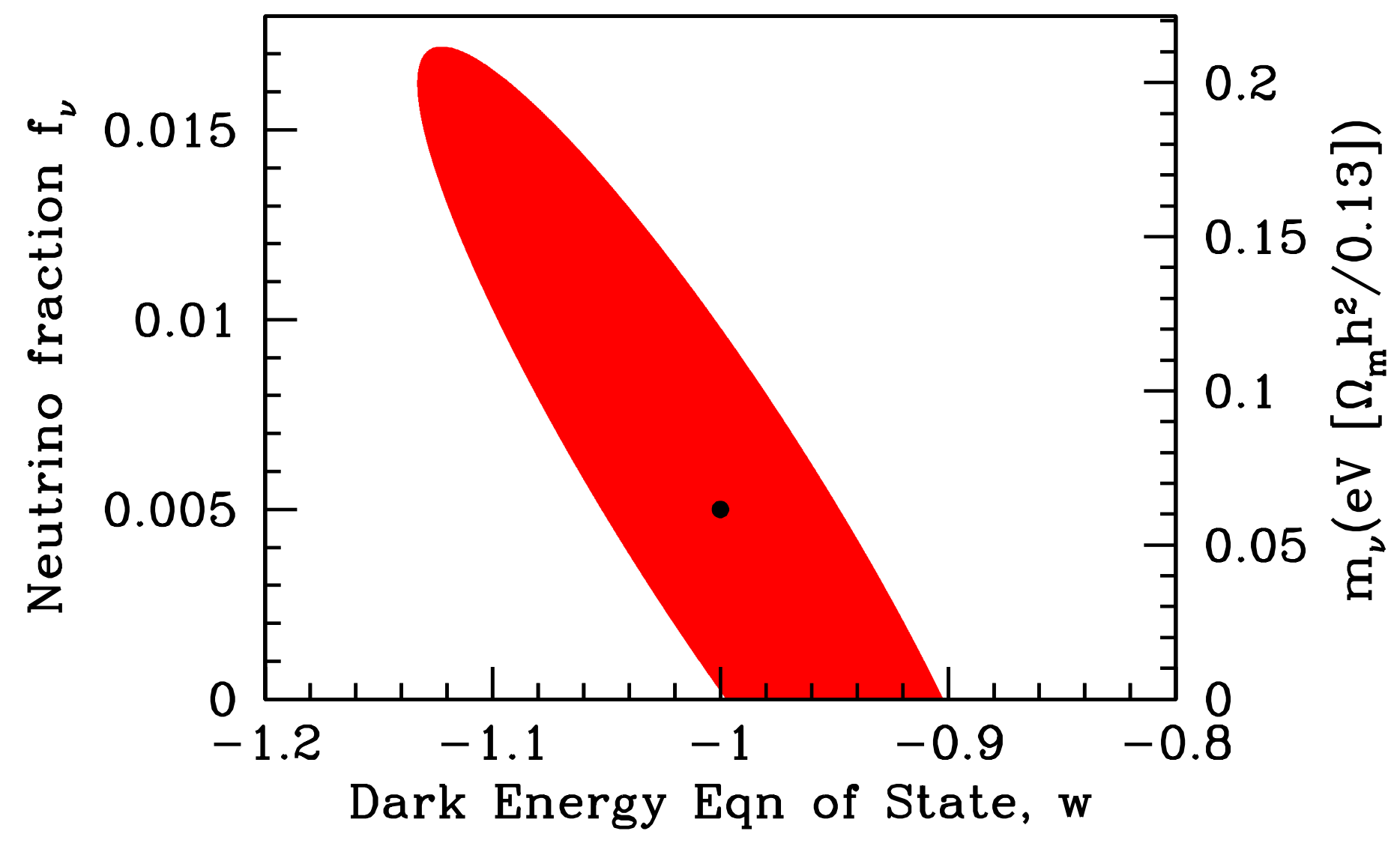

Figure 2. Projected constraints from a survey designed to do weak lensing on a tenth of the sky. Adapted from Ref. [6].

are too small to fulfill all the promise of weak lensing, but it is encouraging to note that cosmic shear currently provides among the best constraints on two cosmological parameters: $\sigma_{8}$ the amplitude of fluctuations and $\Omega_{m}$ the matter density.

The future is bright: a half dozen wide, deep galaxy surveys are planned, and there is little doubt that we will one day have an all-sky map of the cosmic shear out to high enough redshift to learn some very important things. For example, the Dark Energy Task Force confirmed recently that weak lensing is one of four most promising methods for attacking dark energy. Lensing is sensitive to two effects which depend on the nature of the dark energy. First, the weighting function in equation [1] depends on the redshift-distance relationship. Second, using background galaxies in multiple redshift bands, one can measure the evolution of the gravitational potential, an evolution which is non-trivial as long as dark energy exists. This power leads to some odd degeneracies. In particular, another cosmological parameter, the neutrino mass, also leads to the decay of the gravitational potential. So, as shown in Fig. 2, the dark energy equation of state is degenerate with the neutrino mass. 


\section{Beyond the Two-Point Function}

Having stressed the similarity between cosmic shear and the temperature of the CMB, we now turn to a very important difference. The measured CMB is Gaussian. To some extent this is not surprising: anisotropies in the CMB are small; any deviation from Gaussianity in the form of a non-zero three-point function, e.g., would necessarily be down by a factor of $10^{-5}$. This is not true of inhomogeneities in the matter distribution. After decoupling, these inhomogeneities grow. After all, this is the essence of gravitational instability. So, even though the matter distribution starts off as Gaussian with mean zero, the distribution becomes skewed due to gravity. It must be skewed because the smallest value of the density is zero, while the largest is many orders of magnitude larger than the mean.

This leads to one of the most pressing questions in gravitational lensing: is there important information contained beyond the two-point function (there would not be if the distribution was purely Gaussian), and if so how can we extract it?

There are two complementary approaches to this problem. First, one can proceed systematically. We've computed the 2-point function (the power spectrum) and projected what can be learned from it. We can move on to the 3-point function (the bispectrum) and 4-point function (the trispectrum), etc. and see what can be learned from them. A completely different approach involves identifying big things (this is called cluster counting in optical astronomy), or more technically, regions in which the shear is large. This second approach is powerful because the number of clusters as a function of redshift depends sensitively on the underlying cosmology. Given a theory, a cosmologist can predict how many clusters there should be in a given mass and redshift range. So for this approach to work, we need to be able to (i) identify clusters and (ii) determine their masses.

A consensus is emerging that looking at the cosmic shear field is not a good way to find clusters. Rather, we can probably find clusters more efficiently with standard optical or X-ray techniques or even with surveys of the Sunyaev-Zel'dovich effect. Lensing is more powerful for the second purpose: once we have found a cluster, we need a way of determining its mass. In principle lensing offers a direct way of measuring masses without going through an intermediary such as the X-Ray temperature or the optical richness.

\section{CMB Lensing by Clusters}

Measuring a foreground cluster's mass by observing distortions in the shapes of background galaxies has a long history. Newer is the notion that we might be able to use the CMB as the background "source", and from this estimate the mass of foreground clusters $[7,8,9,10,11,12,13,14,15]$. Seljak and Zaldarriaga [7] initially studied this and wrote down a very simple formula for the observed temperature field:

$$
\tilde{T}(\vec{\theta})=T(\vec{\theta}+\vec{\alpha}) \simeq T(\vec{\theta})+\frac{\partial T}{\partial \theta_{i}} \alpha_{i}
$$


where $T$ is the unlensed primordial temperature field, $\tilde{T}$ is the lensed field that we observe, $\vec{\theta}$ is the angular position on the sky, and $\vec{\alpha}$ is the deflection due to structure along the line of sight. Seljak and Zaldarriaga noted that there is little structure on small scales in the primordial CMB, so the first term on the right and the coefficient of the second are likely to be quite simple across the field of a cluster. Under these assumptions, Seljak and Zaldarriaga and others have estimated how accurately one could determine cluster masses by measuring the deflection angle $\vec{\alpha}$. Roughly, one would need an experiment with angular resolution of order an arcminute with sensitivity of order a few microKelvin. Since a number of such experiments are in the planning stages (for other reasons), it makes sense to look carefully at how well we really can do on cluster masses. To do this, we employ a maximum likelihood technique (which we describe in Section 5) on simulated maps of the lensed CMB.

\subsection{Simulations of the lensed $C M B$}

The methods we use are described more fully elsewhere [11], so we provide only a brief outline here. We first generate unlensed CMB temperature anisotropies as a random realization of a Gaussian field with a power spectrum computed using CMBfast [16]. To make lensed maps, we must first know the mass distribution, which we model using an N-body simulation to create a sample distribution of dark matter which we expect to be statistically similar to that of our universe. The simulation uses a TreePM code [17] to evolve structure in a periodic cube $300 \mathrm{Mpc} / \mathrm{h}$ on a side, which is large enough to ensure a fair sample of clusters relevent for our purposes, and employs $512^{3}$ dark matter particles of mass $1.7 \times 10^{10} \mathrm{M}_{\odot} / \mathrm{h}$, so that even the smallest of our clusters comprise thousands of particles, and are sufficiently well resolved for our needs. The cluster's shapes are less regular (and hopefully more realistic) than a simple fit to a spherical profile, and they are located in their appropriate cosmological context, which inlcudes lots of other structure when it is projected to make lensing maps. Since the simulation is dark matter only, it neglects all forces except gravity. This simplifiction allows us to simulate many clusters at reasonable cost, although they are likely to be somewhat less accurate than more sophisticated methods.

The effect of lensing is computed from maps of the convergence, $\kappa$, assuming the weak lensing approximation, which is adequate in this case. The convergence is computed from the density contrast along the past lightcone

$$
\kappa=\frac{3}{2} \Omega_{m} H_{0}^{2} \int d \chi g(\chi) \delta(\chi)
$$

where $\chi$ is the comoving distance, $\delta$ is the density contrast, $\Omega_{m}$ and $H_{0}$ are the present day matter and Hubble parameters, and $g(\chi)$ is a geometrical factor. From this, the deflection angle $\vec{\alpha}$ is obtained

$$
\vec{\alpha}=\nabla\left(\nabla^{-2} \kappa\right)
$$

and the lensed temperature field is then computed using Equation 2. 
Figure 3 shows a simulated region of CMB sky lensed by a massive cluster. The dimple introduced by lensing is not visible in the picture but it is clear that the approximation of a very simple CMB field is not exactly accurate. So here we present an analysis method which accounts for this complication.

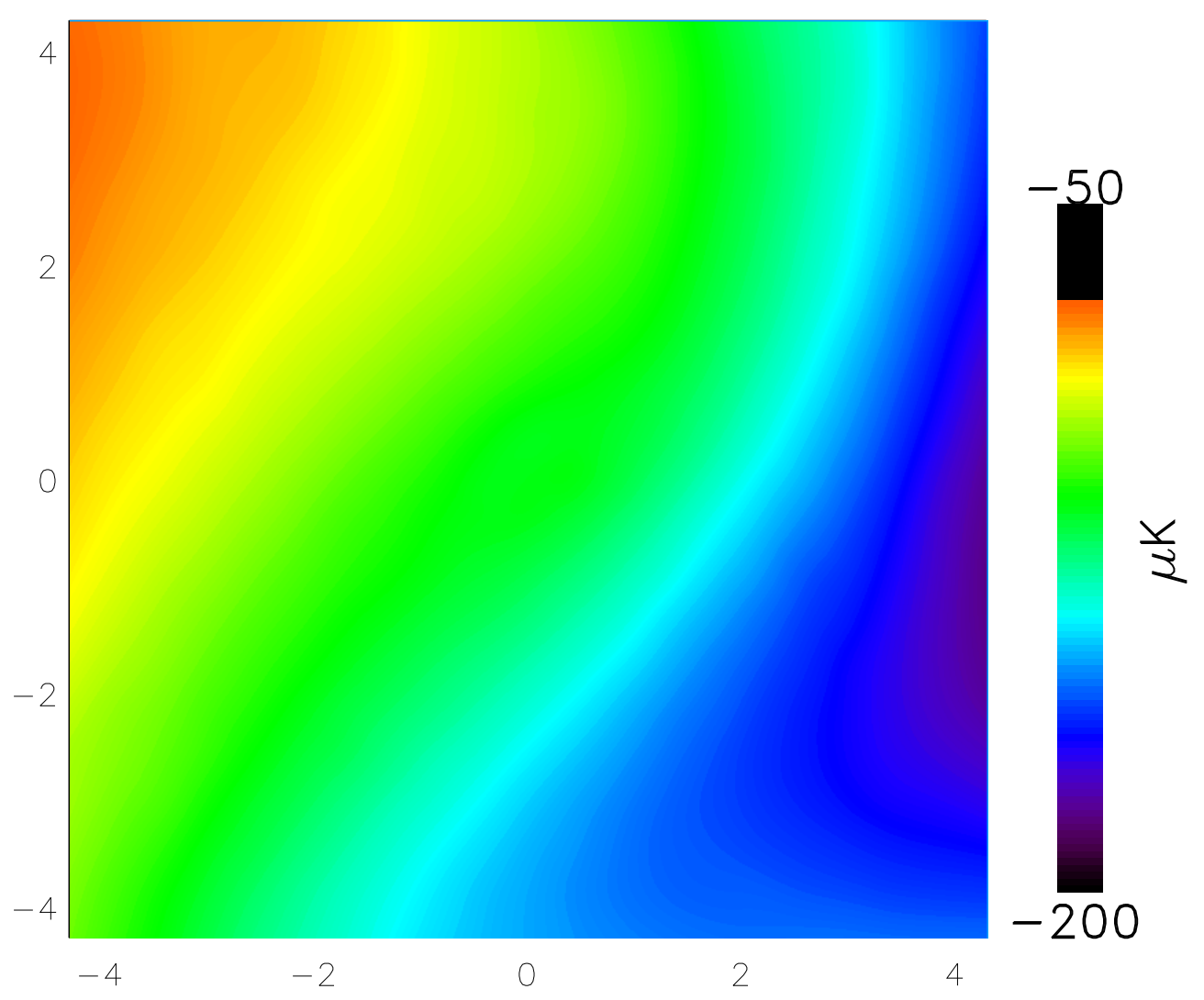

Figure 3. Simulated CMB field behind a cluster at redshift 0.5 with mass $5.6 \times$ $10^{14} h^{-1} M_{\odot}$.

\section{Likelihood estimator}

Several estimators have emerged in the literature $[18,19,20,21]$ which are aimed at recovering the large scale mass distribution using CMB lensing, while others aim to estimate the mass of large local distortions such as clusters [7, 12, 14]. Here we present one of the latter variety. The likelihood function for observing a set of CMB temperatures $\tilde{T}$ in $N$ pixels is Gaussian:

$$
\mathcal{L}=\frac{(2 \pi)^{-N / 2}}{\operatorname{det}(C)^{1 / 2}} \exp \left\{-\frac{1}{2} \tilde{T}_{i} C_{i j}^{-1} \tilde{T}_{j}\right\} .
$$


Here the covariance matrix can be obtained by noting that

$$
\tilde{T}_{i} \simeq T_{i}+\vec{\nabla} T_{i} \cdot \vec{\alpha}_{i}+\frac{1}{2} \frac{\partial^{2} T_{i}}{\partial \theta^{a} \partial \theta^{b}} \alpha_{i}^{a} \alpha_{i}^{b}
$$

To compute the covariance matrix, let's first rewrite the lensed temperature in pixel $i$ as a linear combination of the unlensed temperature in all pixels:

$$
\tilde{T}_{i}=\Lambda_{i j} T_{j}
$$

with

$$
\Lambda_{i j} \equiv \delta_{i j}+\alpha_{i}^{a} \nabla_{i j}^{a}+\frac{1}{2} \alpha_{i}^{a} \alpha_{i}^{b} \Delta_{i j}^{a b} \quad \text { no sum on } i .
$$

The index $a$ runs over the 2 transverse dimensions. The $\nabla$ operator can be written explicitly given a pixelization scheme. For example, for the pixelization shown in Fig. 4, $\nabla_{21}^{1}=-1 / 2 \Delta, \nabla_{23}^{1}=+1 / 2 \Delta$ and $\nabla_{2 j}^{1}=0$ for all other $j$. Similarly, $\nabla_{25}^{2}=-1 / 2 \Delta, \nabla_{24}^{2}=+1 / 2 \Delta$ and $\nabla_{2 j}^{2}=0$ for all other $j$. Meanwhile $\Delta^{a b}$ is the second derivative operator; e.g.,

$$
\Delta_{i j}^{x x}=\frac{1}{4 \Delta^{2}} \begin{cases}1 & j=i+2 \\ -2 & j=i \\ 1 & j=i-2\end{cases}
$$

and zero for all other $j$. More generally, $\Delta_{i j}^{a b}=\nabla_{i i^{\prime}}^{a} \nabla_{i^{\prime} j}^{b}$. Armed with this notation, we immediately see that

$$
C_{i j}=\Lambda_{i i^{\prime}} C_{i^{\prime} j^{\prime}}^{u} \Lambda_{j j^{\prime}}+C_{i j}^{N}
$$

where $C^{u}$ is the covariance matrix of the unlensed CMB and $C^{N}$ includes, e.g., instrumental noise.

As it stands, the covariance matrix, and hence the likelihood function, depends on the cluster profile via its impact on the deflection angle $\vec{\alpha}$. We will adpot a parametric approach, assuming that a cluster at redshift $z$ has an NFW profile, parametrized by its mass and concentration. That is, $\vec{\alpha}$ depends on the mass $m_{200}$ and concentration c. Other structures along the line of sight also affect the deflection angle though. We will assume that these other structures are random with values drawn from a Gaussian distribution, so that

$$
\vec{\alpha}_{i}=\vec{\alpha}_{i}^{\mathrm{NFW}}+\vec{\alpha}_{i}^{\mathrm{lss}}
$$

The last term is the deflection angle due to large scale structure along the line of sight not associated with the cluster.

The covariance matrix therefore depends on a random variable at each pixel, $\vec{\alpha}_{i}^{\mathrm{lss}}$. The correct thing to do would be to integrate these random variables weighted by their distribution functions, $P\left(\vec{\alpha}^{\text {res }}\right)$, for example. The likelihood then would be

$$
\mathcal{L}=\left[\prod_{j} \int d \vec{\alpha}_{j}^{\mathrm{lss}}\right] \mathcal{L}\left(\vec{\alpha}^{\mathrm{lss}}\right) .
$$


Pixel Size $\Delta$

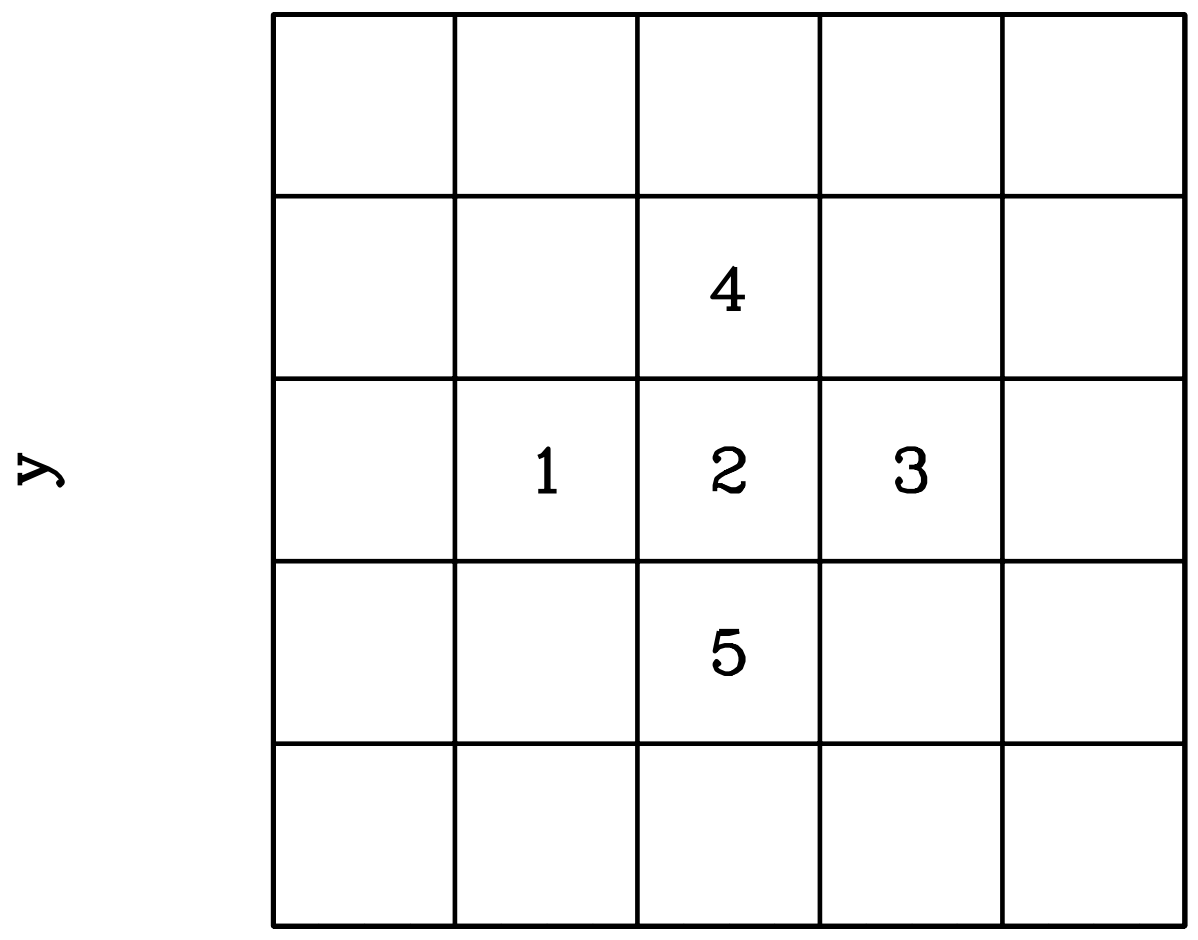

\section{$\mathbf{X}$}

Figure 4. Pixelization scheme. Observed temperature at pixel 2 depends on temperature at adjacent pixels because of gravitational lensing.

This however is an $N^{2}$-dimensional integral, so we need to find approximations. One possible approximation is to let

$$
C\left(\vec{\alpha}^{\mathrm{res}}, \vec{\alpha}^{\mathrm{lss}}\right) \rightarrow\left\langle C\left(\vec{\alpha}^{\mathrm{res}}, \vec{\alpha}^{\mathrm{lss}}\right)\right\rangle
$$

where the angular brackets denote the same integrals as before over the same probability distribution. This time, though, the integrals become trivial, because they reduce simply to the one-point functions (which vanish) and the two-point functions which can be computed easily. Under this approximation then,

$$
\begin{aligned}
C \rightarrow C_{i j} & =\Lambda_{i i^{\prime}}^{\mathrm{NFW}} C_{i^{\prime} j^{\prime}}^{u} \Lambda_{j j^{\prime}}^{\mathrm{NFW}}+C_{i j}^{N}+C_{i j}^{\mathrm{sss}, a b} \nabla_{i i^{\prime}}^{a} \nabla_{j j^{\prime}}^{b} C_{i^{\prime} j^{\prime}}^{u} \\
& +\frac{1}{2}\left[C_{j j}^{\mathrm{lss}, a b} \Delta_{j j^{\prime}}^{a b} C_{i j^{\prime}}^{u}+(i \leftrightarrow j)\right]
\end{aligned}
$$

with no sum on $i, j$ in the last two terms.

Fig. 5 illustrates one example of projected constraints using the likelihood technique. In this case, the analysis gets the correct result, although with fairly large errors. This confirms what a number of people have suggested about CMB-Cluster lensing: the power of this technique will be to stack clusters. The errors from the $\mathrm{CMB}$ on the mass of a single cluster will be too large to provide useful constraints on 


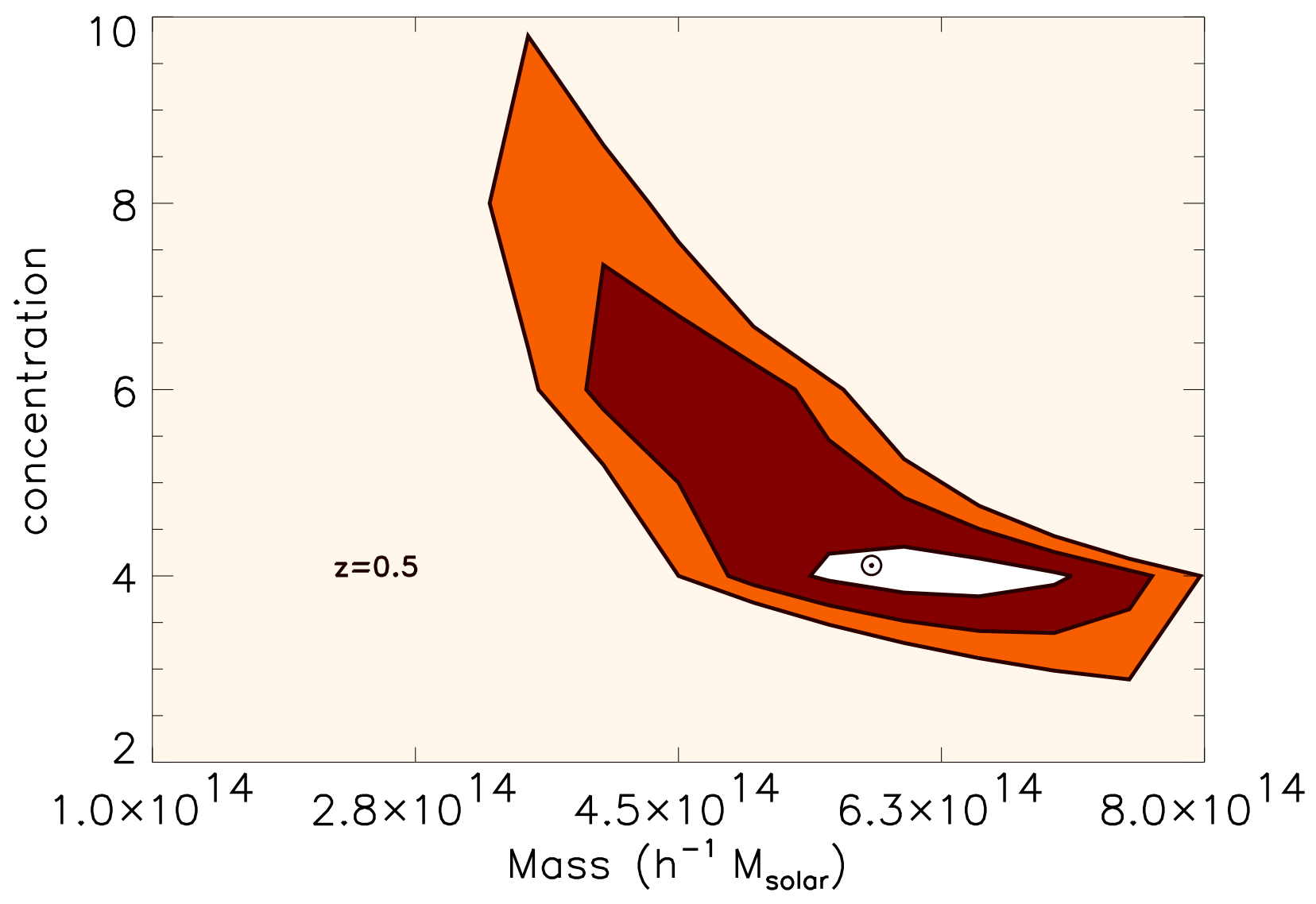

Figure 5. Likelihood contours in mass/concentration space from a $0.5^{\prime}$ pixel CMB experiment with $1 \mu \mathrm{K}$ noise per pixel.

cosmological parameters, but we can combine many clusters in a given bin (say with the same SZ signal) and beat down the errors.

Fig. 6 illustrates a potential problem with this technique: in addition to being noisy, the likelihood estimates are biased. That is, the estimator got the right answer for the cluster in Fig. 5, but in general it does not get the right answer. The average mass estimate is a litte more than $20 \%$ smaller than the true mass, and there are large tails. The shape of this bias is similar to the shape of the distribution function of large scale structure: low peak but high tails. Given our faulty approximation in equation [14], it is not surprising that this bias emerges. An important problem will be to introduce more sophisticated estimators to address this bias.

Indeed there is even more work remaining that fixing the bias. In this work, we have analyzed maps which are free from the kinetic SZ effect. When we analyze those maps using this technique, we get results which are much less impressive. So the likelihood estimator needs to evolve to handle data from upcoming experiments. 


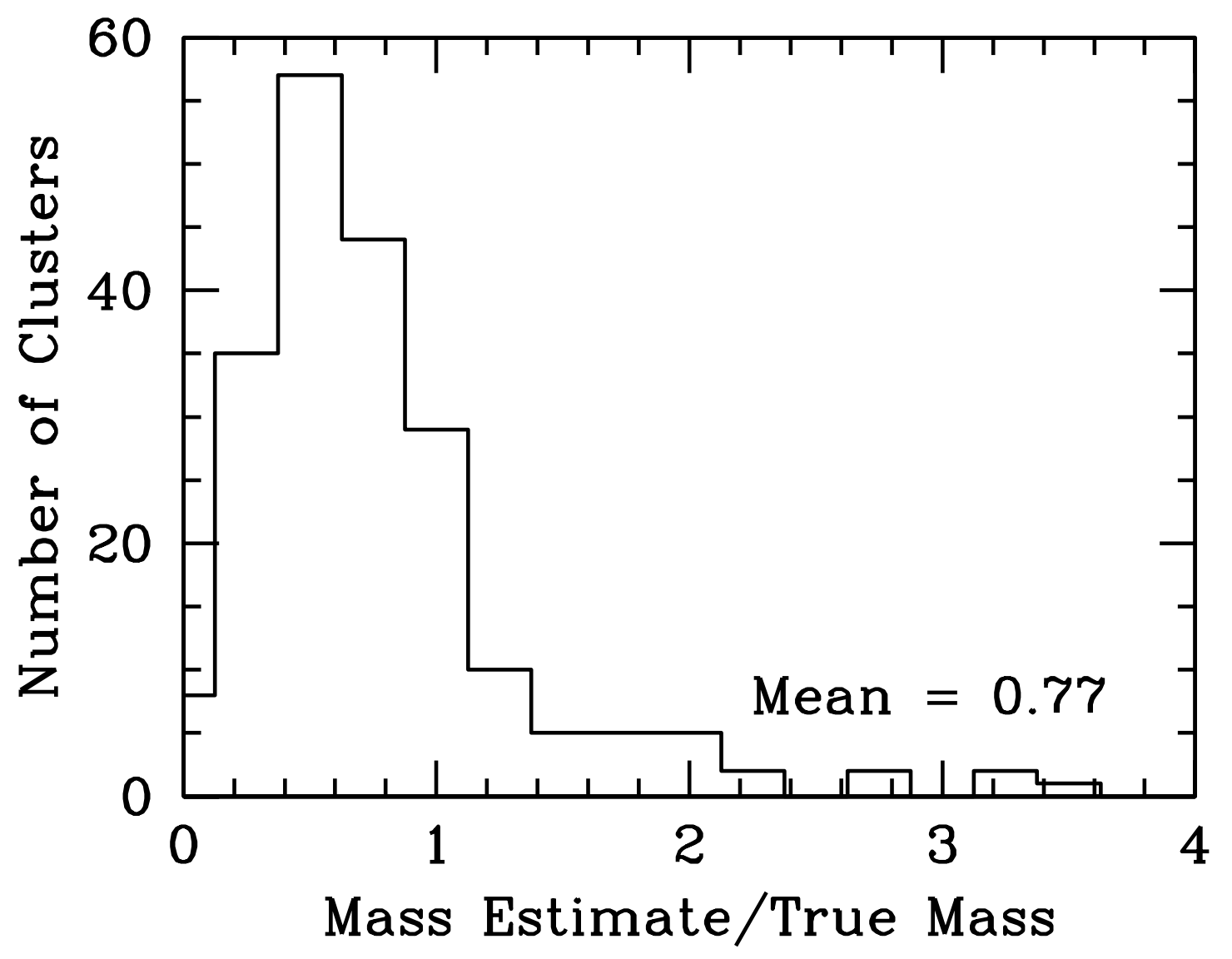

Figure 6. Histogram of the ratio of the cluster mass estimate from CMB to the true cluster mass.

\section{Conclusions}

Gravitational lensing is likely to be a powerful tool for probing cosmology over the next decade. Here, we have focused on one potential application: cluster mass determination from fine-scale measurements of the CMB. Our analysis suggests that this technique has a lot of potential but sophisticated algorithms will be needed to extract the relevant information.

SD would like to thank Joan Sola and the other organizers of IRGAC 2006 for an interesting conference. This work is supported by the US Department of Energy and by NASA grant NAG5-10842.

\section{Appendix A. Large Scale Structure Noise}

Compute $C_{i j}^{\mathrm{lss}, a b}$. The deflection angle is an integral along the line of sight, so using the Limber approximation, we can immediately write down its power spectrum:

$$
C^{\mathrm{lss}, a b}(\vec{l})=-l^{a} l^{b} \int_{0}^{\infty} d \chi \frac{W^{2}(\chi)}{\chi^{4}} P_{\Phi}(l / \chi, \chi) .
$$


The covariance matrix is the Fourier transform of this:

$$
C_{i j}^{\mathrm{lss}, a b}=-\int \frac{d^{2} l}{(2 \pi)^{2}} e^{i \vec{l} \cdot \vec{\theta}_{i j}} l^{a} l^{b} \int_{0}^{\infty} d \chi \frac{W^{2}(\chi)}{\chi^{4}} P_{\Phi}(l / \chi, \chi) .
$$

Rewrite the vectors $l^{a}$ and $l^{b}$ as derivatives with respect to $\theta$. Then, the polar integral becomes simply

$$
\int_{0}^{2 \pi} d \phi e^{i l \theta_{i j} \cos \phi}=2 \pi J_{0}\left(l \theta_{i j}\right) .
$$

So the covariance matrix is

$$
C_{i j}^{\mathrm{lss}, a b}=\frac{\partial^{2}}{\partial \theta^{a} \partial \theta^{b}} \int_{0}^{\infty} \frac{d l l}{(2 \pi)} J_{0}(l \theta) \int_{0}^{\infty} d \chi \frac{W^{2}(\chi)}{\chi^{4}} P_{\Phi}(l / \chi, \chi) .
$$

We can carry out the derivatives here using properties of Bessel functions:

$$
\begin{aligned}
\frac{\partial^{2}}{\partial \theta^{a} \partial \theta^{b}} J_{0}(l \theta) & =-\frac{\partial}{\partial \theta^{a}}\left[\frac{\partial(l \theta)}{\partial \theta^{b}} J_{1}(l \theta)\right] \\
& =-l \frac{\partial}{\partial \theta^{a}}\left[\frac{\theta^{b}}{\theta} J_{1}(l \theta)\right] \\
& =-l \frac{\delta_{a b} J_{1}(l \theta)}{\theta}-l^{4} \theta^{a} \theta^{b}\left\{\frac{1}{x} \frac{d}{d x}\left[\frac{J_{1}(x)}{x}\right]\right\} \\
& =-l \frac{\delta_{a b} J_{1}(l \theta)}{\theta}+l^{2} \theta^{a} \theta^{b}\left\{\frac{J_{2}(l \theta)}{\theta^{2}}\right\} .
\end{aligned}
$$

So we finally have for the covariance matrix

$$
C_{i j}^{\mathrm{lss}, a b}=-\delta^{a b} I_{1}\left(\theta_{i j}\right)+\frac{\theta_{i j}^{a} \theta_{i j}^{b}}{\theta_{i j}^{2}} I_{2}\left(\theta_{i j}\right)
$$

with the two integrals defined as

$$
\begin{aligned}
& I_{1}(\theta) \equiv \frac{1}{\theta} \int_{0}^{\infty} \frac{d l l^{2}}{2 \pi} J_{1}(l \theta) \int_{0}^{\infty} d \chi \frac{W^{2}(\chi)}{\chi^{4}} P_{\Phi}(l / \chi, \chi) \\
& I_{2}(\theta) \equiv \int_{0}^{\infty} \frac{d l l^{3}}{2 \pi} J_{2}(l \theta) \int_{0}^{\infty} d \chi \frac{W^{2}(\chi)}{\chi^{4}} P_{\Phi}(l / \chi, \chi) .
\end{aligned}
$$

The two integrals are shown in Fig. A1. 


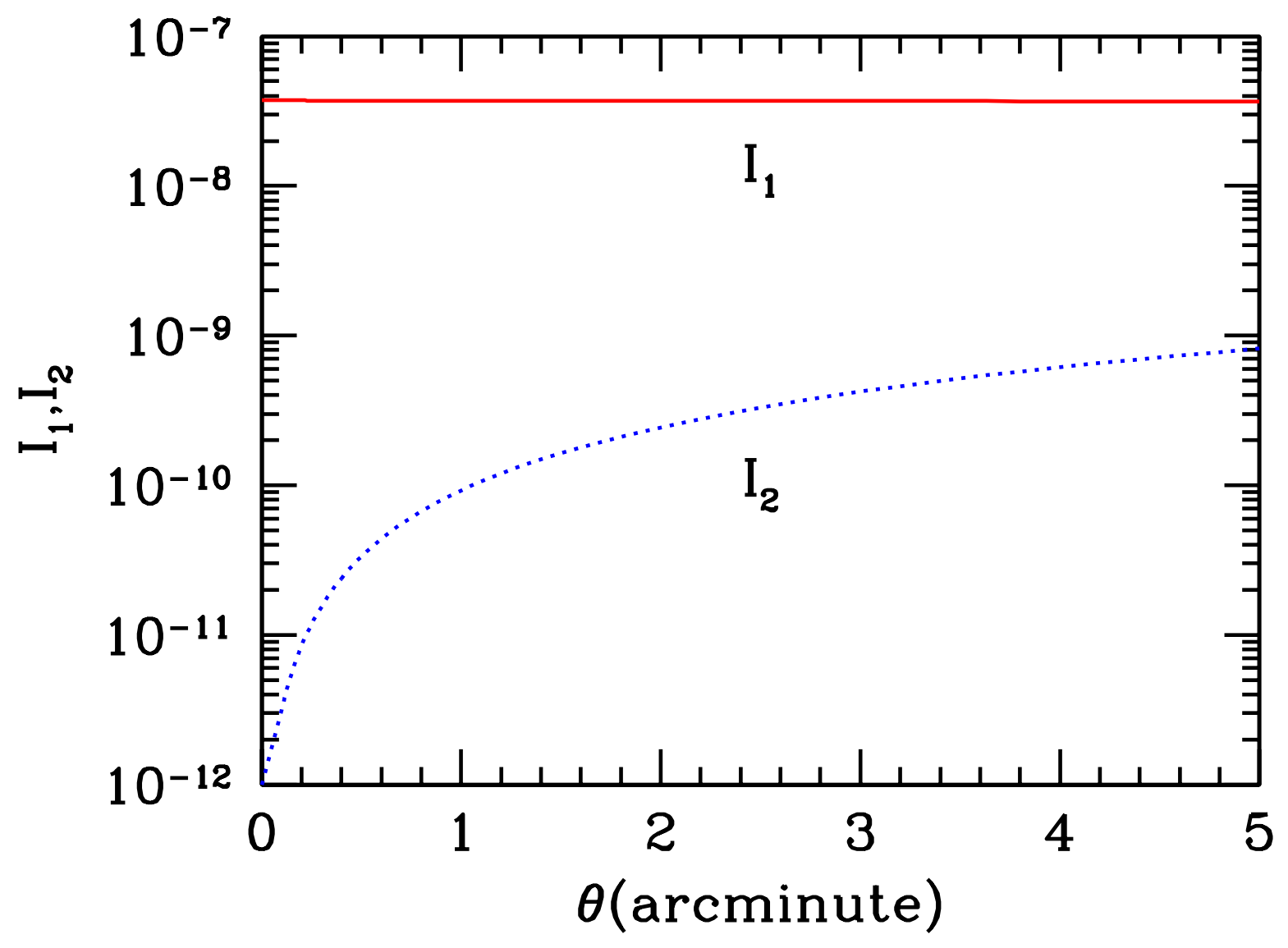

Figure A1. The two integrals in equation [A.7].

\section{References}

[1] Bartelmann M and Schneider P 2001 Phys. Rep. 340 291-472 (Preprint astro-ph/9912508)

[2] Dodelson S 2003 Modern cosmology (Modern cosmology / Scott Dodelson. Amsterdam (Netherlands): Academic Press. ISBN 0-12-219141-2, 2003, XIII + 440 p.)

[3] Lewis A and Challinor A 2006 Phys. Rep. 429 1-65 (Preprint astro-ph/0601594)

[4] Dodelson S, Shapiro C and White M J 2006 Phys. Rev. D73 023009 (Preprint astro-ph/0508296)

[5] Hoekstra H, Yee H and Gladders M 2002 New Astron. Rev. 46 767-781 (Preprint astro-ph/0205205)

[6] Abazajian K N and Dodelson S 2003 Phys. Rev. Lett. 91041301 (Preprint astro-ph/0212216)

[7] Seljak U and Zaldarriaga M 2000 Astrophys. J 538 57-64 (Preprint astro-ph/9907254)

[8] Nagai D, Kravtsov A V and Kosowsky A 2003 Astrophys. J. 587 524-532 (Preprint astro-ph/0208308)

[9] Dodelson S 2004 Phys. Rev. D 70 023009-+ (Preprint astro-ph/0402314)

[10] Holder G P and Kosowsky A 2004 Astrophys. J. 616 8-15 (Preprint astro-ph/0401519)

[11] Vale C, Amblard A and White M 2004 New Astronomy 10 1-15 (Preprint astro-ph/0402004)

[12] Maturi M, Bartelmann M, Meneghetti M and Moscardini L 2005 Astron. Astrophys. 436 37-46 (Preprint astro-ph/0408064)

[13] Lewis A and King L 2006 Phys. Rev. D 73 063006-+ (Preprint astro-ph/0512104)

[14] Hu W, DeDeo S and Vale C 2007 (Preprint astro-ph/0701276)

[15] Vale C, De Deo S and Hu W 2007 in preparation

[16] Seljak U and Zaldarriaga M 1996 Astrophys. J 469 437-+ (Preprint astro-ph/9603033)

[17] White M 2002 Astrophys. J Supp. 143 241-255 (Preprint astro-ph/0207185) 
[18] Zaldarriaga M 2000 Phys. Rev. D 62063510 (Preprint astro-ph/9910498)

[19] Hu W 2001 Astrophys. J Lett. 557 L79 (Preprint astro-ph/0105424)

[20] Hirata C M and Seljak U 2003 Phys. Rev. D67 043001 (Preprint astro-ph/0209489)

[21] Okamoto T and Hu W 2003 Phys. Rev. D 67083002 (Preprint astro-ph/0301031) 